The dynamics and rheology of a dilute suspension of periodically forced neutrally buoyant spherical particles in a quiescent Newtonian fluid at low Reynolds numbers

This article has been downloaded from IOPscience. Please scroll down to see the full text article.

2011 Fluid Dyn. Res. 43045502

(http://iopscience.iop.org/1873-7005/43/4/045502)

View the table of contents for this issue, or go to the journal homepage for more

Download details:

IP Address: 202.41.64.20

The article was downloaded on 30/06/2011 at 04:37

Please note that terms and conditions apply. 


\title{
The dynamics and rheology of a dilute suspension of periodically forced neutrally buoyant spherical particles in a quiescent Newtonian fluid at low Reynolds numbers
}

\author{
T R Ramamohan ${ }^{1}$, I S Shivakumara ${ }^{2}$ and K Madhukar ${ }^{1,2}$ \\ ${ }^{1}$ CSIR Centre for Mathematical Modelling and Computer Simulation (C-MMACS), Council of \\ Scientific and Industrial Research, Wind Tunnel Road, Bangalore-560 037, India \\ ${ }^{2}$ UGC Centre for Advanced Studies in Fluid Mechanics, Department of Mathematics, Bangalore \\ University, Bangalore-560 001, India \\ E-mail: trr@cmmacs.ernet.in, shivakumarais@gmail.com and k.madhukara@gmail.com
}

Received 25 March 2011, in final form 3 June 2011

Published 29 June 2011

Online at stacks.iop.org/FDR/43/045502

Communicated by M Maxey

\begin{abstract}
We study the effects of both convective and unsteady inertia on the dynamics and rheology of a dilute suspension of periodically forced neutrally buoyant spherical particles, at low Reynolds numbers, in a quiescent Newtonian fluid. The inclusion of inertia results in additional terms in the equation governing the dynamics of the particle that represent a fading memory of the entire history of the particle motion. The inclusion of convective inertia in the low Reynolds number limit makes the memory term nonlinear. Several tests were performed to show that the results presented in this paper are physically reasonable and correct. A perturbation analysis of the problem yields strong evidence that the results of our simulations are correct. It is observed that there is a preferred direction in this system which manifests itself in the properties of the solution. This preferred direction is identified as the direction of the initial motion of the particle. We present here results on the behavior of various parameters with respect to Reynolds numbers and the amplitude of the periodic force. These include phase-space plots between particle displacement and particle velocity and the variation of a rheological parameter, namely a 'normal stress' with respect to Reynolds number and the amplitude of the periodic force. We believe that our results may be technologically important since the rheological parameter depends strongly on controllable parameters such as the Reynolds number and the amplitude of the periodic force. Further, this system is one of the simplest systems whose rheology shows non-Newtonian behavior, such as the presence of a normal stress. In addition, this system represents a physically realizable system for experimentally testing the frameworks developed to calculate the collective behavior of systems of oscillators with memory.
\end{abstract}




\section{Introduction}

The flow due to a spherical body moving in a viscous, incompressible fluid at small Reynolds number has been a problem of both practical and theoretical importance. The translation of a rigid sphere was first considered by Stokes (1851), motivated by his interest in the effects of fluid friction on the motion of pendulums. Lovalenti and Brady (1993) have summarized relevant studies conducted prior to 1993 and have also derived an expression for the hydrodynamic force on a rigid particle undergoing arbitrary time-dependent motion at small Reynolds numbers, using the reciprocal theorem. Leshansky and Brady (2004) have calculated the hydrodynamic force on a sphere using the generalized reciprocal theorem considering only unsteady inertia. The effect of inertia on the sedimentation of a sphere near a plane wall has been studied by Becker et al (1996). Jansons (2007) studied the effect of inertia on stochastic Stokes' drift. The influence of fluid inertia on the motion of a finite assemblage of solid spherical particles in a slowly changing uniform flow at small Reynolds number Re and moderate Strouhal number $S l$ has been studied by Leshansky et al (2004). A simulation of flow around spheres and cylinders at finite Reynolds numbers was carried out by Kim and Phillips (2004). The effects of the history force on an oscillating rigid sphere at low Reynolds numbers was studied by Abbad and Souhar (2004). The stationary shear flow around fixed and free bodies at finite Reynolds numbers was studied by Mikulencak and Morris (2004). Roscoe worked on the rheology of a suspension of viscoelastic spheres in a viscous liquid (Roscoe 1967). The unsteady flow about a sphere at low to moderate Reynolds numbers with oscillatory motion was studied by Chang and Maxey (1994). In addition, the study of the dynamics and rheology of a suspension of periodically forced particles in a sheared Newtonian fluid has gained importance for over a decade because of its fundamental consequences and its potential technological implications. The main results pertaining to this area have been summarized in a review by Asokan et al (2005). The system considered is an ideal physical system for testing the effects of periodic forcing at the level of individual particles on rheological parameters at a macroscopic level. Since the rheological parameters are obtained by suitable averages over all the individual particles, this system is an ideal system to study the fundamentally and technologically important question of the conditions under which an average over a large number of individually chaotically varying particles itself varies chaotically. The work summarized by Asokan et al (2005) has provided some partial answers to this question. In the case of dilute suspensions, the periodic forcing appears both in the equations of the dynamics of the individual particles and in the equations of the rheological properties. Thus, there is a nonlinear coupling between the microscopic level of the individual particle and the macroscopic level of the rheological parameters and hence chaos at the microlevel can lead to chaos at the macrolevel of the rheological parameters. It has also been demonstrated that fluctuations in a suspension of sheared spheres, including the effects of multiple hydrodynamic interactions simulated by the Stokesian dynamics technique are governed by a low dimensional chaotic attractor (Kumar and Ramamohan 1995). This suggests that chaos at the level of the individual particles can also lead to chaos at the level of the averages when hydrodynamic interactions are included. All of the above results were obtained at zero Reynolds number or by complete neglect of particle and fluid inertia. The aim of the present paper is to take the first step towards extending these results to the regime of low Reynolds numbers. The main impact of inertia in the low Reynolds number regime is to introduce a lag between the imposition of the force and the response of the particle. Mathematically this results in the equation containing terms representing a fading memory of the entire history of the particle motion (Kumar et al 1998, Radhakrishnan et al 1999, Dasan et al 2002). 
If we include convective inertia in the problem (in addition to unsteady inertia), even at low Reynolds numbers, we find that the character of the fading memory term changes and it becomes nonlinear. As a first step in considering the effect of these terms on the dynamics and rheology of periodically forced suspensions of particles, we study the dynamics and rheology of a dilute suspension of periodically forced spherical particles in a quiescent Newtonian fluid at low Reynolds number. The problem considered here and the results obtained herein may also serve as a test of more advanced models when we develop the area further. Further, our analysis leads to the development of an expression for a macroscopic, experimentally realizable parameter from the equations for the dynamics of an individual particle and thus may serve as a test for the techniques of averaging that relate the rheology of a suspension to the dynamics of individual particles with memory.

In the next section, we present the governing equations of the problem. In section 3 , we formulate the problem by using the hydrodynamic force expression for a spherical particle given by Lovalenti and Brady (1993). In section 4, the methodology and tests performed for the consistency of the software are given, and the results are discussed in section 5. In the last section, we conclude with a brief summary of the main results.

\section{Governing equation}

The problem is governed by the full Navier-Stokes equations

$$
\begin{aligned}
& -\nabla p^{\prime}+\mu \nabla^{2} \boldsymbol{u}^{\prime}=\rho\left(\frac{\partial \boldsymbol{u}^{\prime}}{\partial t}+\boldsymbol{u}^{\prime} \cdot \nabla \boldsymbol{u}^{\prime}\right), \\
& \nabla \cdot \boldsymbol{u}^{\prime}=0
\end{aligned}
$$

with the boundary conditions

$$
\begin{aligned}
& \boldsymbol{u}^{\prime}=\boldsymbol{U}_{\mathbf{p}}(\boldsymbol{t}) \quad \text { on the surface of the particle, } \\
& \boldsymbol{u}^{\prime} \rightarrow \boldsymbol{U}^{\infty}(\boldsymbol{t}), \quad p^{\prime} \rightarrow p^{\infty} \quad \text { as } \quad\left|\boldsymbol{x}-\boldsymbol{Y}_{\mathbf{p}}(\boldsymbol{t})\right| \rightarrow \infty
\end{aligned}
$$

Here, $\boldsymbol{U}^{\infty}(\boldsymbol{t})$ is the uniform undistributed flow far from the particle and $\boldsymbol{Y}_{\mathbf{p}}(\boldsymbol{t})$ is the position vector of the centre of mass of the particle. The pressure $p^{\infty}$ satisfies

$$
-\nabla p^{\infty}=\rho \dot{\boldsymbol{U}}^{\infty}(t)
$$

For our purposes, it is more convenient to consider the problem in a translating coordinate system with the origin at the instantaneous centre of the particle. Thus assuming $\boldsymbol{r}=\boldsymbol{x}-$ $\boldsymbol{Y}_{\mathbf{p}}(\boldsymbol{t}), \boldsymbol{u}=\boldsymbol{u}^{\prime}-\boldsymbol{U}^{\infty}(\boldsymbol{t})$ and $p=p^{\prime}-p^{\infty}$, the problem becomes in dimensionless form

$$
\begin{aligned}
& -\nabla p+\nabla^{2} \boldsymbol{u}=\operatorname{Re} S l \frac{\partial \boldsymbol{u}}{\partial t}+\operatorname{Re} \boldsymbol{u} \cdot \nabla \boldsymbol{u}-\operatorname{Re} \boldsymbol{U}_{\mathbf{s}}(t) \cdot \nabla \boldsymbol{u}, \\
& \nabla \cdot \boldsymbol{u}=0
\end{aligned}
$$

with

$$
\begin{aligned}
& \boldsymbol{u}=\boldsymbol{U}_{\mathbf{s}}(\boldsymbol{t}) \quad \text { on the surface of the particle, } \\
& \boldsymbol{u}, p \rightarrow 0, \quad \text { as } \quad|\boldsymbol{r}| \rightarrow \infty \\
& -\nabla p^{\infty}=\operatorname{Re} \operatorname{Sl} \dot{\boldsymbol{U}}^{\infty}(\boldsymbol{t})
\end{aligned}
$$


Here, the slip velocity of the particle $\boldsymbol{U}_{\mathrm{s}}(\boldsymbol{t})=\boldsymbol{U}_{\mathrm{p}}(\boldsymbol{t})-\boldsymbol{U}^{\infty}(\boldsymbol{t})$. The Reynolds number $\operatorname{Re}=$ $a U_{\mathrm{c}} / v$ and the Strouhal number is $S l=\left(a / U_{\mathrm{c}}\right) / \tau_{\mathrm{c}}$, where $a, \boldsymbol{U}_{\mathrm{c}}$ and $\tau_{\mathrm{c}}$ are the characteristic particle dimension, particle slip velocity and timescale, respectively. $\boldsymbol{U}_{\mathbf{s}}(t)$ and $\boldsymbol{u}$ have been non-dimensionalized by $\boldsymbol{U}_{\mathrm{c}}, \boldsymbol{r}$ by $a$ and $p$ by $\mu \boldsymbol{U}_{\mathrm{c}} / a$. $\boldsymbol{u}$ remains bounded for all time, $-\infty<t<\infty$.

\section{Formulation of the problem}

The Lovalenti and Brady (1993) formalism for the hydrodynamic force on a rigid sphere undergoing arbitrary time-dependent motion in an arbitrary time-dependent uniform flow field at small Reynolds numbers is given by the following expression:

$$
\begin{aligned}
F^{\boldsymbol{H}}(t)=\frac{4 \pi}{3} & R e S l \dot{\boldsymbol{U}}^{\infty}(t)-6 \pi \boldsymbol{U}_{\mathbf{s}}(t)-\frac{2 \pi}{3} \operatorname{ReSl} \dot{\boldsymbol{U}}_{\mathbf{s}}(t)+\frac{3}{8}\left(\frac{\operatorname{Re} S l}{\pi}\right)^{1 / 2} \\
\times & \left\{\int _ { - \infty } ^ { t } \left[\frac{2}{3} \boldsymbol{F}_{\mathbf{s}}^{\boldsymbol{H}_{\|}}(t)-\left\{\frac{1}{|\boldsymbol{A}|^{2}}\left(\frac{\pi^{1 / 2}}{2|\boldsymbol{A}|} \operatorname{erf}(|\boldsymbol{A}|)-\exp \left(-|\boldsymbol{A}|^{2}\right)\right)\right\} F_{\mathbf{s}} \boldsymbol{H}_{\|}(s)\right.\right. \\
+ & \left.\frac{2}{3} F_{\mathbf{s}}^{\boldsymbol{H}_{\perp}}(t)-\left\{\exp \left(-|\boldsymbol{A}|^{2}\right)-\frac{1}{2|\boldsymbol{A}|^{2}}\left(\frac{\pi^{1 / 2}}{2|\boldsymbol{A}|} \operatorname{erf}(|\boldsymbol{A}|)-\exp \left(-|\boldsymbol{A}|^{2}\right)\right)\right\} F_{\mathbf{s}}^{\boldsymbol{H}_{\perp}}\right] \\
\times & \left.\frac{2 \mathrm{~d} s}{(t-s)^{3 / 2}}\right\}+\mathrm{O}(\operatorname{Re}) .
\end{aligned}
$$

This expression is obtained by using the reciprocal theorem. The details of the derivation can be found in the work by Leshansky et al (2004). Here, $\boldsymbol{U}_{\mathbf{s}}=\boldsymbol{U}_{\mathbf{p}}-\boldsymbol{U}^{\infty}$ is the slip velocity of the fluid; $\boldsymbol{U}_{\mathbf{p}}$ is the velocity of the particle; and $\boldsymbol{U}_{\mathbf{s}}$ has been non-dimensionalized by $U_{\mathrm{c}}$. The acceleration terms $\dot{\boldsymbol{U}}_{\mathrm{s}}$ and $\dot{\boldsymbol{U}}^{\infty}$ are non-dimensionalized by $\boldsymbol{U}_{\mathrm{c}} / \tau_{\mathrm{c}}$, where $\tau_{\mathrm{c}}$ is the characteristic timescale; $U^{\infty}$ is the velocity of the fluid as $|r| \rightarrow \infty$; Re is the Reynolds number, defined as $R e=a U_{\mathrm{c}} / v$ based on a characteristic slip velocity, $\boldsymbol{U}_{\mathrm{c}} ; a$ denotes the characteristic particle dimension; and $v$ is the kinematic viscosity of the fluid. $\boldsymbol{F}_{\mathbf{s}}^{\boldsymbol{H}_{\|}}=-6 \pi \boldsymbol{U}_{\mathbf{s}}$. $\underline{\boldsymbol{p} \boldsymbol{p}}$ and $\boldsymbol{F}_{\mathrm{s}}^{\boldsymbol{H}_{\perp}}=-6 \pi \boldsymbol{U}_{\mathrm{s}} \cdot(\delta-\underline{\boldsymbol{p p}})$, where $\delta$ is the same tensor of order 2 and unit vector

$$
\underline{\boldsymbol{p}}=\frac{\boldsymbol{Y}_{\mathbf{S}}(t)-\boldsymbol{Y}_{\mathbf{S}}(s)}{\left|\boldsymbol{Y}_{\mathbf{s}}(t)-\boldsymbol{Y}_{\mathbf{S}}(s)\right|},
$$

where $\boldsymbol{Y}_{\mathbf{S}}(t)-\boldsymbol{Y}_{\mathbf{S}}(s)$ is the integrated displacement of the particle relative to the fluid from time $s$ to the current time $t$. Note that $S l$ is the Strouhal number and

$$
\boldsymbol{A}=\frac{\operatorname{Re}}{2}\left(\frac{t-s}{\operatorname{Re} S l}\right)^{1 / 2}\left(\frac{\boldsymbol{Y}_{\mathbf{s}}(t)-Y_{\mathbf{s}}(s)}{t-s}\right) \cdot \boldsymbol{F}^{H}
$$

is scaled by $\mu a U_{\mathrm{c}}$.

For this problem, we consider a neutrally buoyant spherical particle in an infinite body of quiescent fluid and consider the effects of an external periodic force acting on the sphere along the $x$-axis as shown in figure 1 .

We use equation (3.1) to obtain the equation governing the unidirectional motion of a sphere in a quiescent fluid, starting with zero velocity at time $t=0$, with $\boldsymbol{U}_{\mathbf{s}}=\boldsymbol{U}_{\mathbf{p}}$ where $\boldsymbol{U}_{\mathbf{p}}$ is the velocity of the particle, scaled with respect to the size of the particle and the frequency of the external periodic force, $\omega$, i.e. we take $\boldsymbol{U}_{\mathrm{c}}=a \omega$ and $\boldsymbol{U}^{\infty}=0$. Under these conditions, 


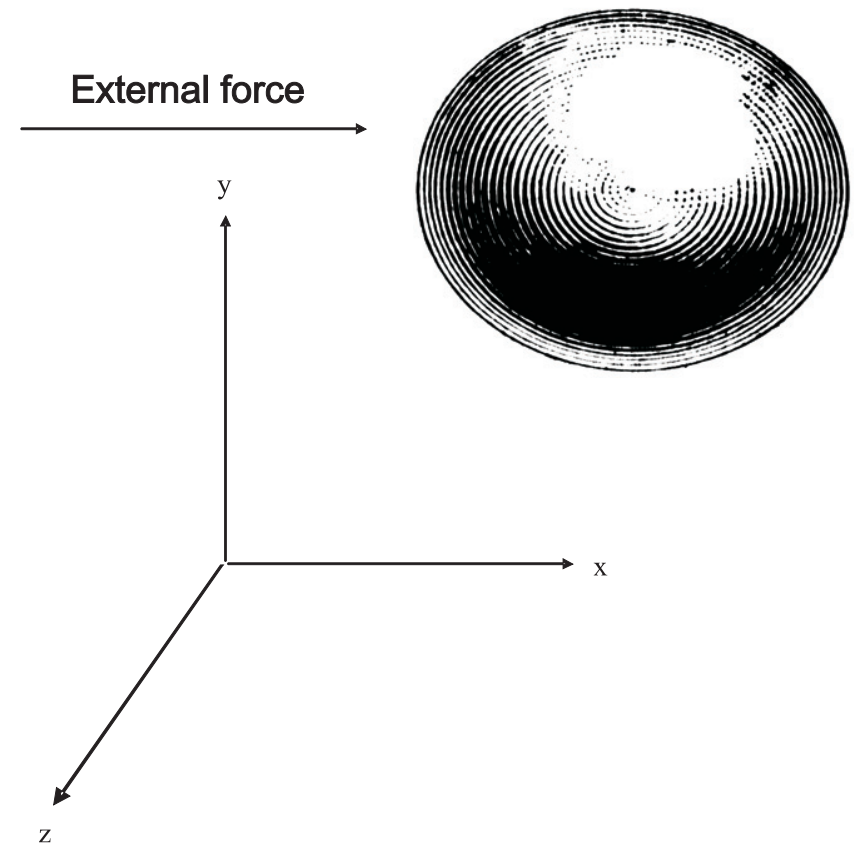

Figure 1. A neutrally buoyant spherical particle in a quiescent Newtonian fluid, on which an external periodic force is applied along the $x$-axis.

equation (3.1) reduces to

$$
\begin{aligned}
\boldsymbol{F}^{\boldsymbol{H}}(t)=-6 \pi \boldsymbol{U}_{\mathbf{p}}(t)-\frac{2 \pi}{3} \operatorname{ReSl} \dot{\boldsymbol{U}}_{\mathbf{p}}(t)+\frac{3}{8}\left(\frac{\operatorname{Re} S l}{\pi}\right)^{1 / 2} \int_{0}^{t}\left[\frac{-8 \pi \boldsymbol{U}_{\mathbf{p}}(t) \mathrm{d} s}{(t-s)^{3 / 2}}\right. \\
\left.-\left\{\frac{1}{|\boldsymbol{A}|^{2}}\left(\frac{\pi^{1 / 2}}{2|\boldsymbol{A}|} \operatorname{erf}(|\boldsymbol{A}|)-\exp \left(-|\boldsymbol{A}|^{2}\right)\right)\right\} \frac{-12 \pi \boldsymbol{U}_{\mathbf{p}}(s) \mathrm{d} s}{(t-s)^{3 / 2}}\right] .
\end{aligned}
$$

We note that the integral in equation (3.2) contains a singularity at $s=t$. In order to take into account this singularity, the integral was split into the intervals $[0, t-\varepsilon]$ and $[t-\varepsilon, t]$ for a small positive $\varepsilon$. Thus, we obtained two expressions for the integral.

Here,

$$
\boldsymbol{A}=\frac{\operatorname{Re}}{2}\left(\frac{t-s}{\operatorname{Re} S l}\right)^{1 / 2}\left(\frac{\boldsymbol{Y}_{\mathbf{s}}(t)-\boldsymbol{Y}_{\mathbf{s}}(s)}{t-s}\right)
$$

That is, we obtain

$$
\boldsymbol{F}^{\boldsymbol{H}}(t)=-6 \pi \boldsymbol{U}_{\mathbf{p}}(\boldsymbol{t})-\frac{2 \pi}{3} \operatorname{Re} S l \dot{\boldsymbol{U}}_{\mathbf{p}}(\boldsymbol{t})+\frac{3}{8}\left(\frac{\operatorname{Re} S l}{\pi}\right)^{1 / 2}(\boldsymbol{P}+\boldsymbol{Q})
$$

where

$\boldsymbol{P}=\int_{0}^{t-\varepsilon}\left[\frac{-8 \pi \boldsymbol{U}_{\mathbf{p}}(t) \mathrm{d} s}{(t-s)^{3 / 2}}-\left\{\frac{1}{|\boldsymbol{A}|^{2}}\left(\frac{\pi^{1 / 2}}{2|\boldsymbol{A}|} \operatorname{erf}(|\boldsymbol{A}|)-\exp \left(-|\boldsymbol{A}|^{2}\right)\right)\right\} \frac{-12 \pi \boldsymbol{U}_{\mathbf{p}}(s) \mathrm{d} s}{(t-s)^{3 / 2}}\right]$ 
and

$$
\boldsymbol{Q}=\int_{t-\varepsilon}^{t}\left[\frac{-8 \pi \boldsymbol{U}_{\mathbf{p}}(t) \mathrm{d} s}{(t-s)^{3 / 2}}-\left\{\frac{1}{|\boldsymbol{A}|^{2}}\left(\frac{\pi^{1 / 2}}{2|\boldsymbol{A}|} \operatorname{erf}(|\boldsymbol{A}|)-\exp \left(-|\boldsymbol{A}|^{2}\right)\right)\right\} \frac{-12 \pi \boldsymbol{U}_{\mathbf{p}}(s) \mathrm{d} s}{(t-s)^{3 / 2}}\right]
$$

Denoting the components of $\boldsymbol{F}^{\boldsymbol{H}}(t), \boldsymbol{U}_{\mathbf{p}}(t), \dot{U}_{\mathbf{p}}, \boldsymbol{A}, \boldsymbol{P}$ and $\boldsymbol{Q}$ along the direction of the force field by $F^{H}(t), \boldsymbol{U}_{\mathrm{p}}(t), \dot{U}_{\mathrm{p}}, A, P$ and $Q$ and then transforming the integral with respect to $A$, we obtain

$$
Q=\int_{0}^{c \sqrt{\varepsilon}} \frac{8 \pi U_{\mathrm{p}}^{2}(t) \operatorname{Re} \mathrm{d} A}{(\operatorname{Re} S l)^{1 / 2} \boldsymbol{A}^{2}}-\int_{0}^{c \sqrt{\varepsilon}} \frac{1}{\left|A^{2}\right|}\left(\frac{\sqrt{\pi}}{2|A|} \operatorname{erf}(|A|)-\exp \left(-\left|\boldsymbol{A}^{2}\right|\right)\right) \frac{12 \pi U_{\mathrm{p}}^{2}(t) \operatorname{Re} \mathrm{d} A}{(\operatorname{Re} S l)^{1 / 2} \boldsymbol{A}^{2}}
$$

where

$$
c=\frac{\operatorname{Re} U_{\mathrm{p}}(t)}{2 \sqrt{\operatorname{Re} S l}}
$$

Note that $Q$ vanishes as $\varepsilon$ tends to zero; that is, as $s \rightarrow t$,

$$
\frac{1}{\left|A^{2}\right|}\left(\frac{\sqrt{\pi}}{2|A|} \operatorname{erf}(|A|)-\exp \left(-\left|A^{2}\right|\right)\right) \rightarrow \frac{2}{3}
$$

Hence, the two singular terms cancel each other as $s \rightarrow t$, and thus we obtain an expression for the hydrodynamic force on a sphere in a quiescent fluid as

$$
\begin{aligned}
& F^{H}(t)=-6 \pi U_{\mathrm{p}}(t)-\frac{2 \pi}{3} \operatorname{Re} S l \dot{U}_{\mathrm{p}}(t)+\frac{3}{8}\left(\frac{\operatorname{Re} S l}{\pi}\right)^{1 / 2} \\
& \times\left\{\int_{0}^{t-\varepsilon}\left[\left\{\frac{1}{|A|^{2}}\left(\frac{\pi^{1 / 2}}{2|A|} \operatorname{erf}(|A|)-\exp \left(-|A|^{2}\right)\right)\right\} \frac{12 \pi U_{\mathrm{p}}(s) \mathrm{d} s}{(t-s)^{3 / 2}}\right]\right. \\
& \left.+16 \pi U_{\mathrm{p}}(t)\left[\frac{1}{\sqrt{t}}-\frac{1}{\sqrt{\varepsilon}}\right]\right\} \text {. }
\end{aligned}
$$

The equation of motion for a neutrally buoyant particle immersed in a fluid is given by

$$
\frac{\boldsymbol{m}_{\mathrm{p}} \dot{\boldsymbol{U}}_{\mathbf{p}}(t)}{\mu a^{2} \omega}=\boldsymbol{F}^{\mathbf{e x t}}(t)+\boldsymbol{F}^{\boldsymbol{H}}(t)
$$

Using equation (3.4) with the external periodic force $F^{\text {ext }}=F_{0} \sin (t)$, where time has been scaled with respect to the frequency of the external periodic force field, along the $x$-direction, and using Newton's law, we obtain equations for the particle velocity $\boldsymbol{U}_{\mathrm{p}}$ and position $Y_{\mathrm{p}}$ with the velocity and position being equal to zero at $t=0$ in the form

$$
\begin{aligned}
\frac{\mathrm{d} Y_{\mathrm{p}}}{\mathrm{d} t} & =U_{\mathrm{p}} \\
\frac{\mathrm{d} U_{\mathrm{p}}}{\mathrm{d} t} & =\frac{1}{R e^{*}}\left[R e_{F} \sin (t)-6 \pi U_{\mathrm{p}}+\frac{3}{8}\left(\frac{\operatorname{Re} S l}{\pi}\right)^{1 / 2}\left(J_{1}+I_{1}\right)\right] .
\end{aligned}
$$


Here,

$$
\begin{aligned}
& J_{1}=16 \pi U_{\mathrm{p}}(t)\left[\frac{1}{\sqrt{t}}-\frac{1}{\sqrt{\varepsilon}}\right] \text { and } \\
& I_{1}=\int_{0}^{t-\varepsilon}\left\{\frac{1}{A^{2}}\left(\frac{\sqrt{\pi}}{2|A|} \operatorname{erf}(A)-\exp \left(-A^{2}\right)\right)\right\} \frac{12 \pi U_{\mathrm{p}}(s)}{(t-s)^{3 / 2}} \mathrm{~d} s .
\end{aligned}
$$

Note that

$$
R e^{*}=\frac{4 \pi}{3} R e+\frac{2 \pi}{3} R e S l, \quad R e_{\mathrm{F}}=\frac{F_{0}}{\mu a^{2} \omega} \quad \text { and } \quad R e=\frac{\rho a^{2} \omega}{\mu},
$$

where $a$ is the particle size, $\omega$ is the frequency of the applied external periodic force, $\mu$ is the viscosity of the fluid and $\rho$ is the density of the particle and the fluid (since the particle is assumed to be neutrally buoyant).

\section{Methodology}

We developed a program using Numerical Recipes in FORTRAN 77 (Press et al 1992) to solve equations ( $3.5 a)$ and (3.5b) using an embedded Runge-Kutta method with adaptive step size. The integral in equation (3.5b) was evaluated at each time step by Romberg extrapolation. The function with respect to $A$ was defined by a user-supplied function subprogram. We used the ODEINT, RKQS and RKCK subroutines from Numerical Recipes to implement the Runge-Kutta method. The Romberg extrapolation was performed using the QROMB subroutine. The integral was evaluated using TRAPZD and the interpolation during the numerical quadrature was performed by POLINT. The main function calls the ODEINT routine, which in turn calls the RKCK and RKQS routines to implement the Runge-Kutta fourth order method with adaptive step size. The Runge-Kutta method was implemented using the subroutine DERIVS, which contained the two differential equations. The integrals involved in calculating the function in DERIVS were written as a separate function and this integration at each iteration was performed using the QROMB subroutine in DERIVS. The QROMB subroutine called the TRAPZD routine to perform the integration. The tolerance, defined as

(Error in successive iterations)/(Value of the function in the previous step),

for both the Romberg extrapolation and the Runge-Kutta solver was taken as $10^{-5}$. Further reduction of the tolerance did not result in any significant change in our results. The entire program was written in double precision. The initial conditions for both the velocity and the position of the particle were taken as zero. $\varepsilon$ was taken as 0.04 ; smaller values of $\varepsilon$ did not significantly change the results for $R e_{\mathrm{F}}$ greater than approximately 0.01 , but for $R e_{\mathrm{F}}$ $\mathrm{O}(0.01)$ lower values of $\varepsilon$ were needed for numerical errors to be avoided. However, this resulted in a large increase in computational time. The software was tested for consistency by compiling the program with two compilers, namely Intel Fortran and F90. We generated 5000 data points taken at an interval of $\pi / 200$ in both the dimensionless velocity and the dimensionless position. Further increase of the resolution did not lead to any change in our results.

In addition to this, the following tests were performed to validate the simulation.

Test 1: Perturbation analysis

We performed a perturbation analysis of the system of equations (3.5a) and (3.5b). The perturbation parameter in this system was taken to be $R e^{1 / 2}$ and the velocity and displacement 


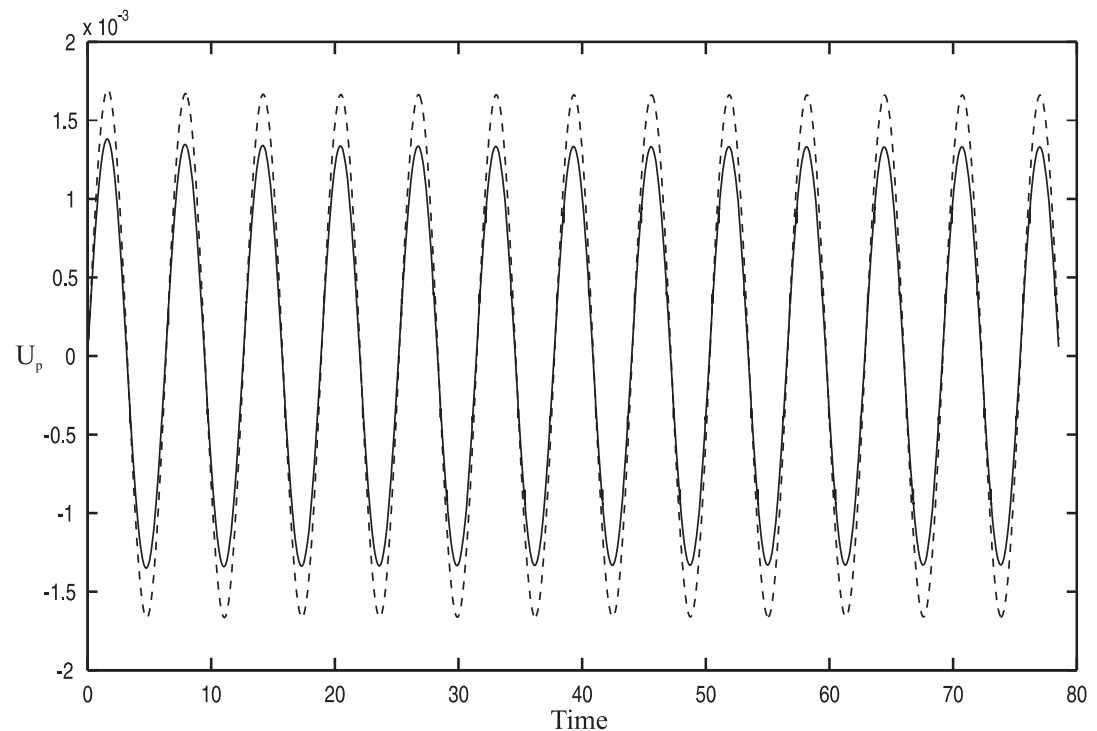

Figure 2. The velocity time series of both the numerical solution (solid line) and the perturbation solution (dashed line) for $R e=0.01$ and $R e_{\mathrm{F}}=0.03$.

of the particle were obtained up to $\mathrm{O}(R e)$.

$U_{0}=\frac{R e_{\mathrm{F}} \sin (t)}{6 \pi} \mathrm{O}(1)$

$U_{1}=\frac{R e_{\mathrm{F}}}{6 \pi}\left(\frac{S l}{\pi}\right)^{1 / 2}\left(\sin (t)\left(\frac{1}{\sqrt{t}}-\frac{1}{\sqrt{\varepsilon}}\right)+\frac{1}{2} \int_{0}^{t-\varepsilon} \frac{\sin (s) \mathrm{d} s}{(t-s)^{3 / 2}}\right) \mathrm{O}\left(R e^{1 / 2}\right)$,

$U_{2}=\frac{1}{6 \pi}\left[\frac{-k R e_{\mathrm{F}} \cos (t)}{6 \pi}+R e_{\mathrm{F}}\left(\frac{S l}{\pi}\right)\left(\sin (t)\left(\frac{1}{\sqrt{t}}-\frac{1}{\sqrt{\varepsilon}}\right)^{2}+\frac{1}{2} \int_{0}^{t-\varepsilon} \frac{\sin (s) \mathrm{d} s}{(t-s)^{3 / 2}}\right.\right.$

$+\frac{1}{2}\left[\int_{0}^{t-\varepsilon}\left\{\frac{\sin (s)}{(t-s)^{3 / 2}}\left(\frac{1}{\sqrt{s}}-\frac{1}{\sqrt{\varepsilon}}\right) \mathrm{d} s\right.\right.$

$\left.\left.\left.\left.+\frac{1}{2} \int_{0}^{s} \frac{\sin \left(s_{1}\right)}{\left(s-s_{1}\right)^{3 / 2}} \mathrm{~d} s_{1} \frac{\mathrm{d} s}{(t-s)^{3 / 2}}\right\}\right]\right)\right] \quad \mathrm{O}(R e)$.

Here,

$$
k=\left(\frac{4 \pi}{3}+\frac{2 \pi S l}{3}\right) .
$$

We obtained the corresponding value of $Y_{\mathrm{p}}$ by solving the differential equation $(3.5 a)$.

A Fortran code and a Matlab script were written in order to obtain the values of $\boldsymbol{U}_{\mathrm{p}}$ and $Y_{\mathrm{p}}$ and these were compared with the time series and phase plots obtained for the full solution, both including the nonlinear term and excluding the nonlinear term. We found that at low values of $R e$ both the perturbation solution and the numerical solution matched (typically up to $R e \approx 0.05)$. Figure 2 presents a comparison of the velocity time series of the full solution with that of the perturbation solution for $R e=0.01$ and $R e_{\mathrm{F}}=0.03$. The differences in the values 
of the numerical solution and perturbation solution such as deviation from the perturbation solution at high acceleration are possibly due to our linear approximation of the nonlinear memory term. Even though $R e$ is small, the parameter $A$ in equation (3.1) need not be $\mathrm{O}(R e)$ even at low Reynolds numbers, for all $t$ and $s$.

Test 2: The solution to the problem of the motion of a spherical particle of greater density than the fluid starting from rest derived using the assumptions of Reynolds number $R e \ll 1$ and the Strouhal number $S l$ known from the literature (figure 5 of Leshansky et al (2004)) was reproduced.

Test 3: The velocity of the fluid at infinity was assumed to be a constant, i.e. $\boldsymbol{U}^{\infty}=\boldsymbol{U}_{0}$, and with $R e_{\mathrm{F}}=0$, it was obtained that $U_{\mathrm{p}} \rightarrow U_{0}$ as $t \rightarrow \infty$, which is as expected.

Test 4: A number of outputs were generated with $\boldsymbol{U}_{0}=0$ and $U_{0} \neq 0$ and the results were compared as $U_{0} \rightarrow 0$. The results matched for $\boldsymbol{U}_{0}=0$ and $U_{0} \rightarrow 0$, up to an order of $10^{-5}$ in the relative fractional error.

Test 5: When the initial direction of the motion was reversed, namely by replacing $R e_{\mathrm{F}}$ with $-R e_{\mathrm{F}}$, the phase-space plot was reflected about the zero velocity axis. That is, a reflection of the phase-space attractor about the zero velocity axis when the direction of the first motion is reversed was obtained, which can be considered as an important result that increases our confidence in our results. The results showed a preferred direction in the solution. Since the only physical direction in our problem is the initial direction of the external force, a reversal of that direction should result in a reversal of direction in the solution, which was indeed the case.

Test 6: We observed that there is a shift in the position of the attractors when we change the initial condition of $Y_{\mathrm{p}}$. Changing $Y_{\mathrm{p}}$ at $t=0$ results in a shift in the position of attractors as there is only a change in the frame of reference, which does not affect the physics of the problem. This confirms that a change in the initial position only results in a change in the coordinate system and not in any physical parameter. This further increases our confidence in our results.

We note here that $\boldsymbol{U}_{\mathrm{p}} \neq 0$ at $t=0$ does not make any physical sense, since if we consider $\boldsymbol{U}_{\mathrm{p}} \neq 0$ at $t=0$, then there must be some particle velocity at negative time too. Moreover, in a quiescent fluid, the particle velocity is due to the application of the external periodic force, which is applied only from $t=0$.

These tests give us considerable confidence in our results.

\section{Results and discussions}

Typical phase-space plots (plots of particle velocity versus particle position) are plotted in figures 3-5 for different values of the Reynolds number $R e$ and the amplitude of the periodic force $R e_{\mathrm{F}}$. These plots represent a bounded region of phase space and hence the plots represent an attractor in phase space. Here, as $R e_{\mathrm{F}}$ increases, the attractor size also increases, establishing the obvious relation between the amplitude $R e_{\mathrm{F}}$ of the forcing term and the size of the attractors. As the amplitude of the periodic force increases, the particle also oscillates with greater amplitude, covering a greater surface area in the attractor plot. One can observe from the two figures, figures 3 and 4, that as $R e$ increases, the area bounded by the attractors decreases, showing the effect of inertia on the motion of the particle. Re represents the magnitude of the inertial term, namely a resistance to change in motion. We also note here that as $R e$ increases, the resistance to the change in motion also increases, diminishing the size of the attractors. 

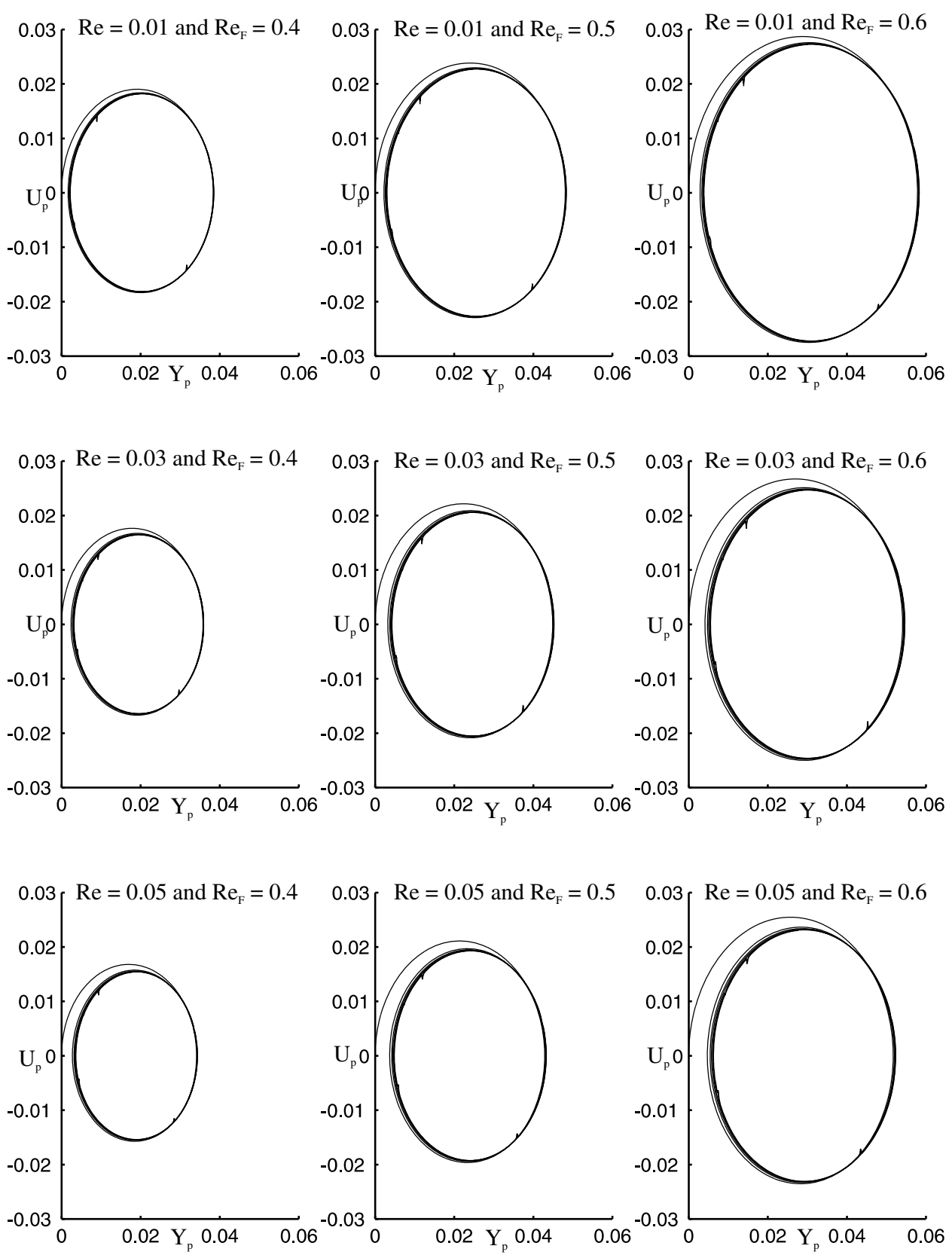

Figure 3. The phase portrait for various low values of $R e$ and high values of $R e_{\mathrm{F}}$. These attractors show the effect of the amplitude of the periodic force, $R e_{\mathrm{F}}$, on the phase plot.

Figure 5 gives an example of the phase plot at $R e=0.5$ and $R e_{\mathrm{F}}=0.01$. This was computed with $\varepsilon=0.004$ in order to avoid numerical errors in the phase plots. The other plots in figures 3 and 4 were generated with a much higher $\varepsilon$, namely $\varepsilon=0.04$. Further reduction in the $\varepsilon$ value did not change the results at higher values of $R e_{\mathrm{F}}$. For $R e_{\mathrm{F}}=0.01$ with $\varepsilon=0.009$, we could avoid numerical errors. However, the generation of figure 5 consumed a 

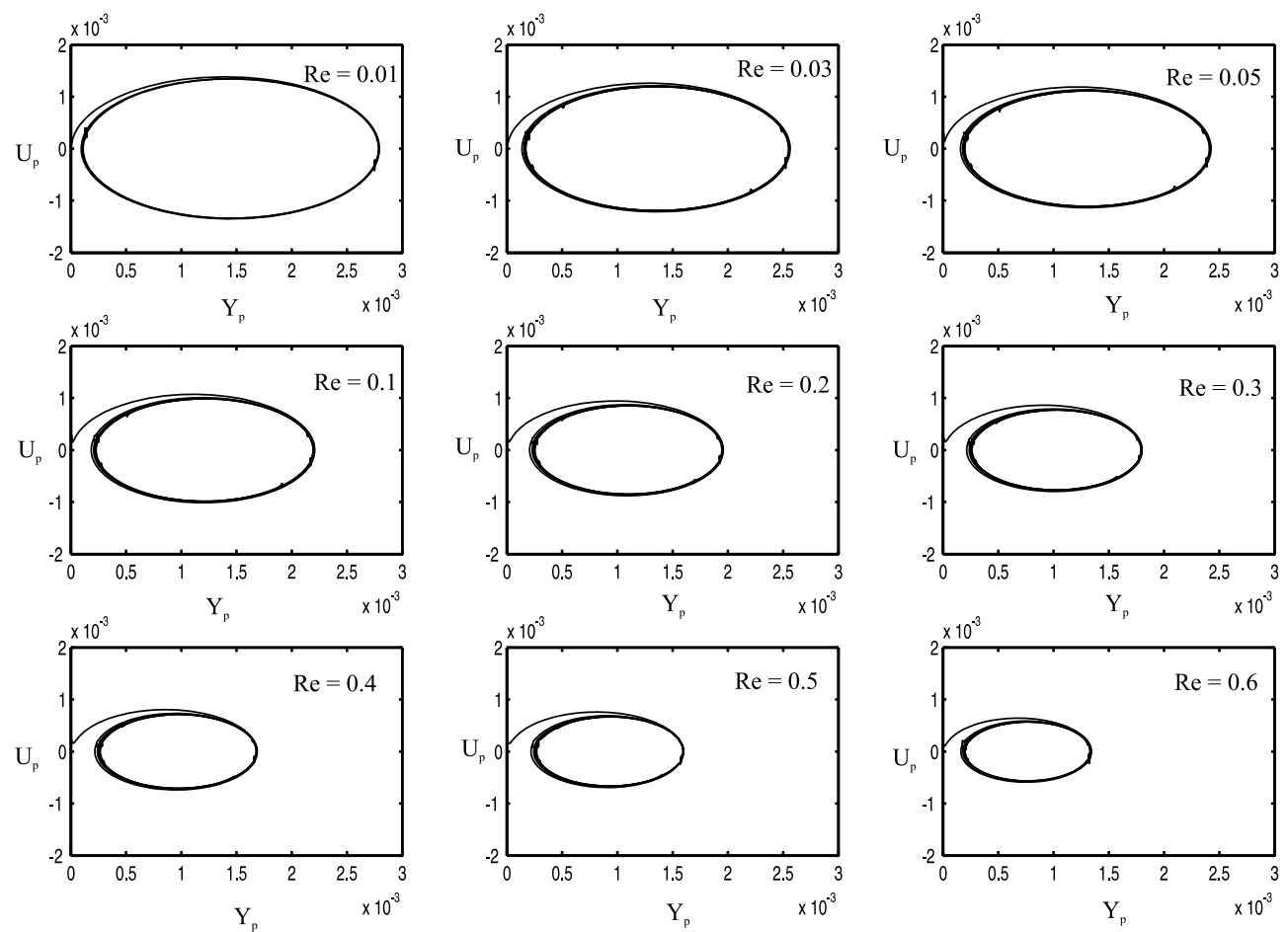

Figure 4. The phase portrait for various values of $R e$ and at $R e_{\mathrm{F}}=0.03$. These attractors show the effect of the Reynolds number $R e$ on the attractors.

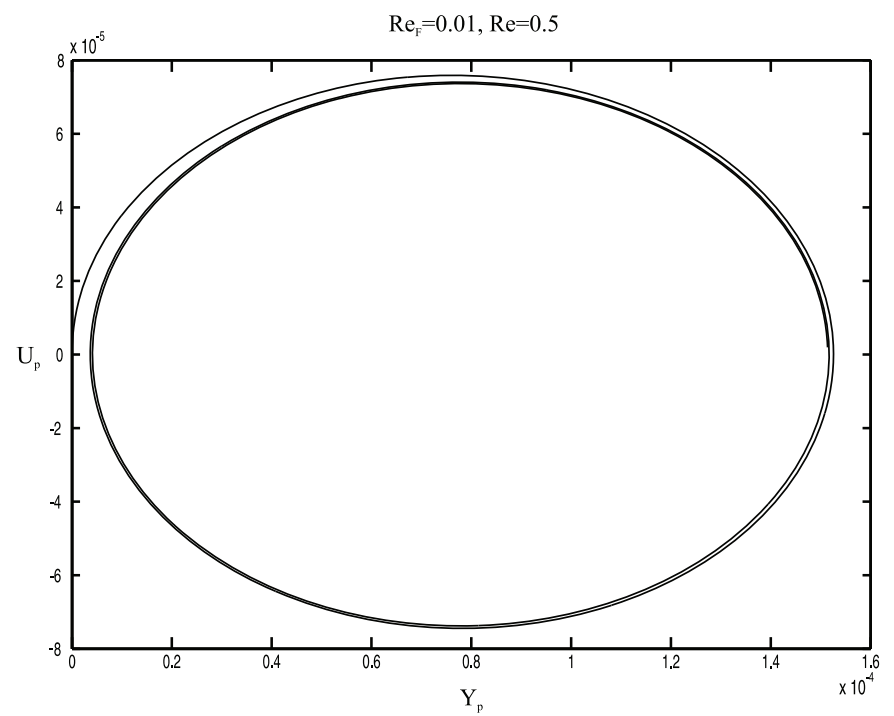

Figure 5. The typical phase portrait for $R e_{\mathrm{F}}=0.01$.

large amount of computational time (around 50 days to compute 5000 lines of data on an SGI Altix350 machine).

Figure 6 shows the power spectrum for different $R e_{\mathrm{F}}$ and for $R e=0.3$. This figure shows that for the values of $R e$ that we have considered, there are no higher harmonics present in the 

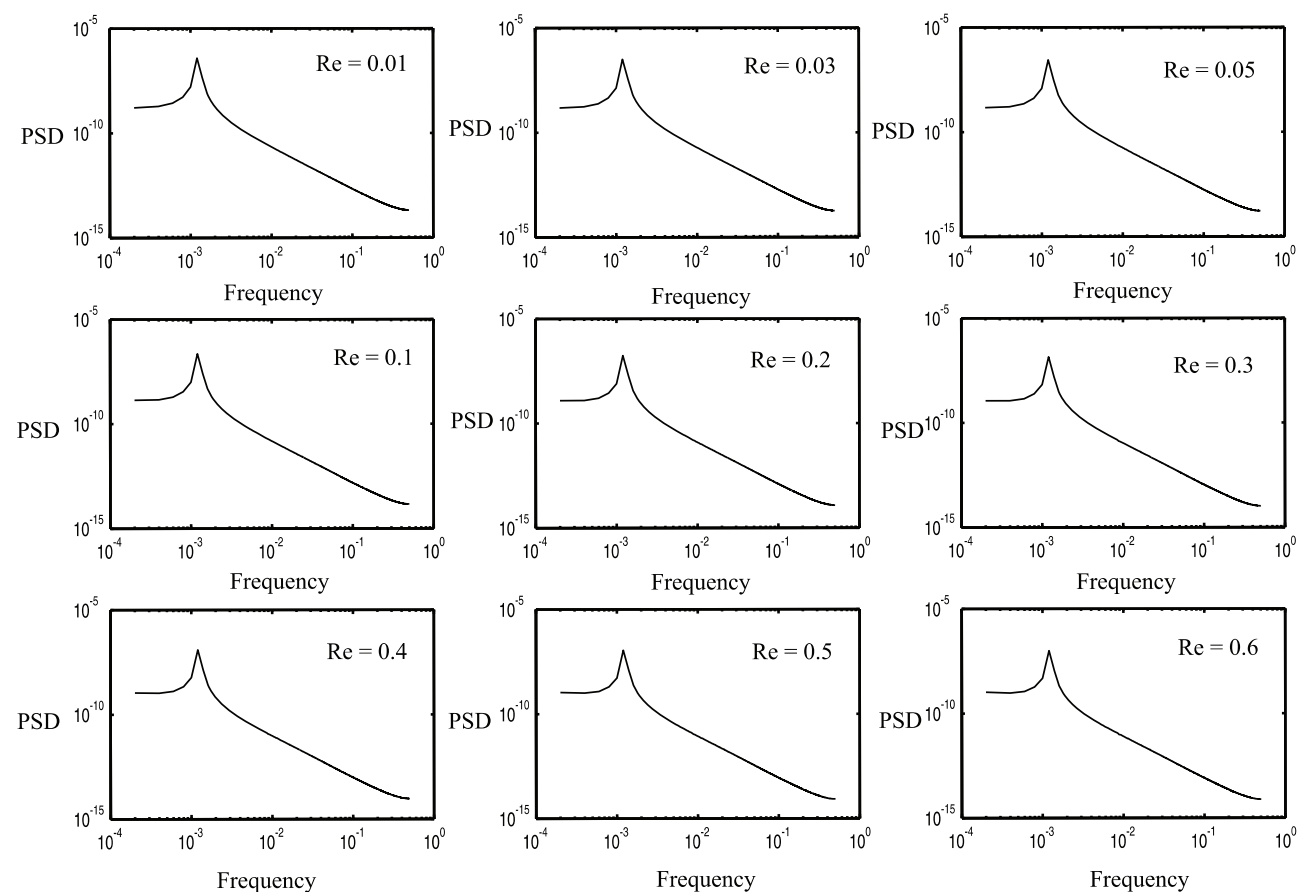

Figure 6. Power spectrum for various values of $R e$ and at $R e_{\mathrm{F}}=0.03$.

time series. We analyzed the behavior of the system by neglecting the nonlinear term and we compared it with the attractors obtained by including the nonlinearity (figures 3-5).

Figure 7 shows the plot of the attractors obtained by setting the nonlinear term to be equal to zero. We observe that the attractors follow a spiral in all cases. There is a slight drift of the particle from its mean position in the long time limit in a particular direction. This direction can be identified as the direction of the first motion of the particle. When we compare figure 7 with figures 3 and 4 , we observe that figures 3 and 4 have greater surface area and there is a greater drift in the initial motion of the particle. This is due to the nonlinearity of the system. In these regions the inertia is high and hence nonlinear effects are also high.

Figures 3, 4 and 7 show that the limit cycle of Stokes' flow is replaced by a slight spiral. This may be due to there being a small net displacement of the particle after every cycle and hence a small drift, resulting in a relatively small spiral motion. The direction of the drift is in the same direction as the direction of first motion. In addition, there is a net displacement of the mean position of the particle from zero. The direction of this displacement is also the same as the direction of first motion, which is the only physical direction in the problem. The numerical solution obtained was compared with the Stokes' flow results. The amplitudes of the time series of the velocity of the particle and of the Stokes' flow case were compared. As $R e$ tends to zero, the amplitudes of both Stokes' flow and the numerical solution approach each other. Figure 8 shows a surface plot of $R e$ and $R e_{\mathrm{F}}$ against the amplitude ratio. Here, the amplitude ratio is the ratio of the amplitude of the velocity of the particle in our problem with a similar velocity for Stokes' flow. We note that the plot shows a decreasing trend. This is due to the effect of inertia of the fluid. As $R e$ increases the amplitude ratio decreases, showing the effect of inertia is to reduce the amplitude of the motion as compared with the Stokes' flow case. Since there is a slight drift of the particle, the relationship of the mean of the 

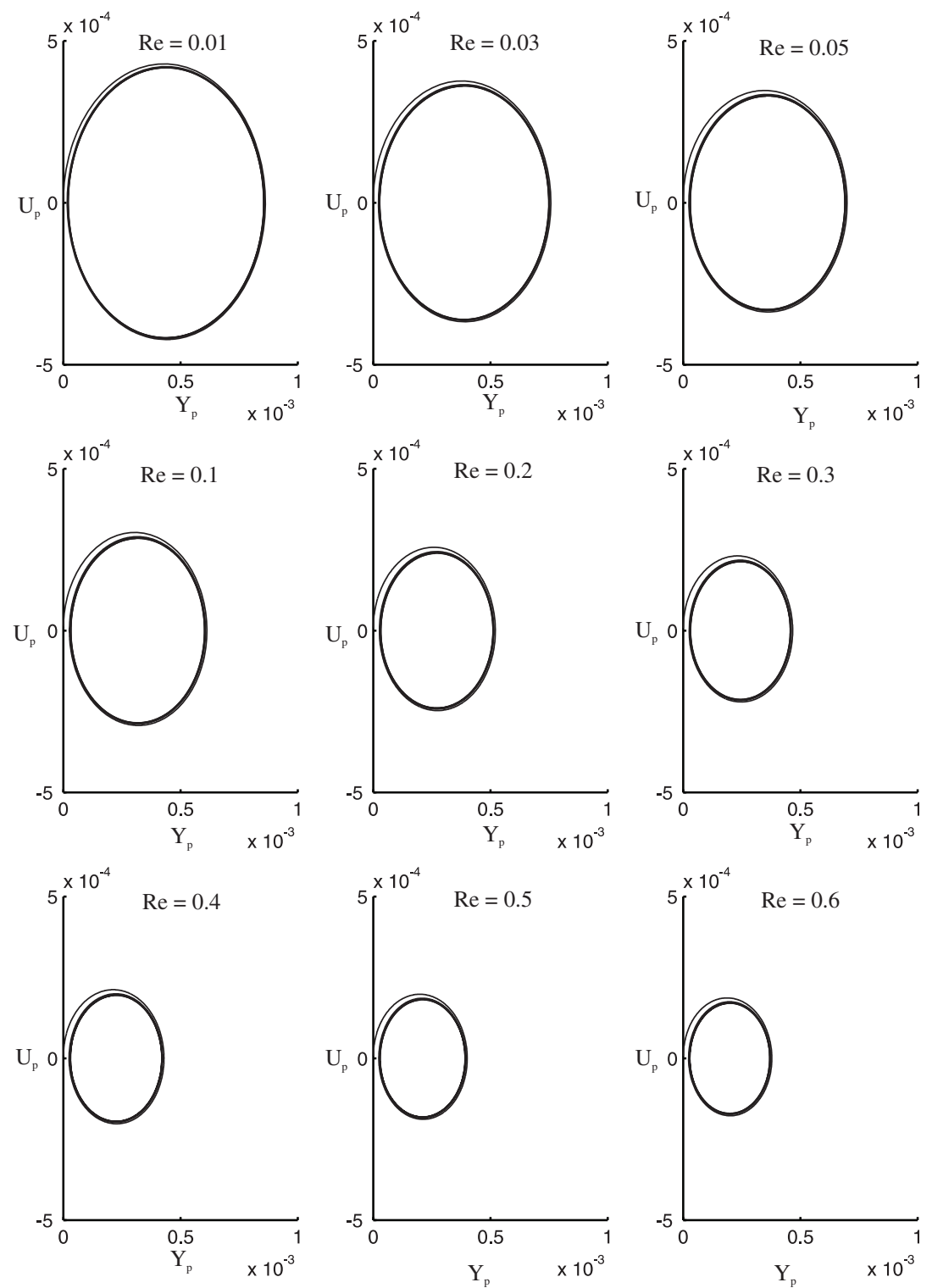

Figure 7. The phase portraits for various $R e$ and $R e_{\mathrm{F}}=0.01$, neglecting the nonlinear term.

displacement of the particle $Y_{\mathrm{p} \text {, mean }}$ (the average position of the particle) with $R e$ and $R e_{\mathrm{F}}$ is obtained and is shown in figure 9. We observed that there is a definite relationship between the mean particle displacement $Y_{\mathrm{p} \text {,mean }}$ and the amplitude of the external periodic force $R e_{\mathrm{F}}$. This is as expected, since the magnitude of the initial motion is determined by the amplitude of the periodic external force and by the value of $R e$. As $R e$ increases we note that $Y_{\mathrm{p} \text {,mean }}$ decreases, and as $\operatorname{Re}_{\mathrm{F}}$ increases $Y_{\mathrm{p} \text {,mean }}$ increases.

When we apply a phase shift of $\pi$ to the sinusoidal forcing term the attractors shift their direction. That is, when we apply a force in an initially negative direction (the opposite direction), $Y_{\mathrm{p}}$ shows a reflection about the $Y_{\mathrm{p}}=0$ axis and thus we obtain a reflection of the 


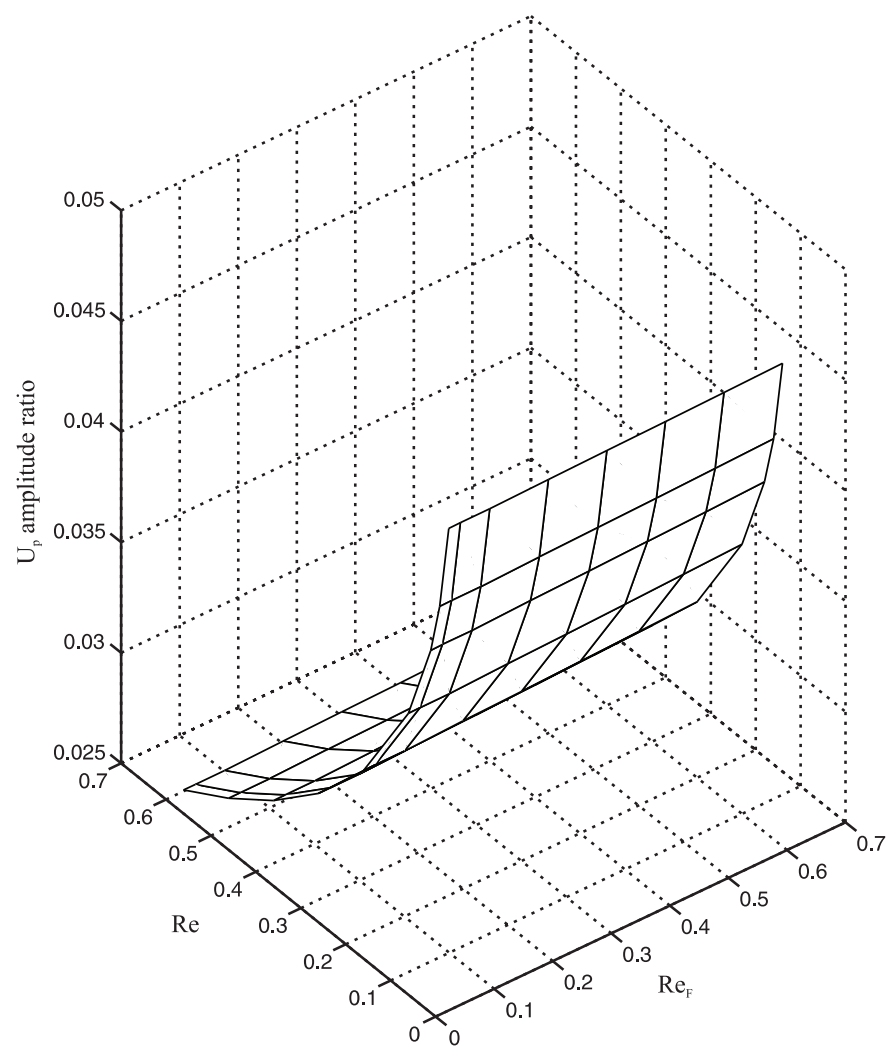

Figure 8. A plot of the amplitude ratio against $R e$ and $R e_{\mathrm{F}}$. We note that as $R e$ increases, the amplitude ratio decreases.

attractor. Figure 10 shows the phase plots when the direction of the amplitude of the force is changed and the attractors form a reflection of each other about the axis $Y_{\mathrm{p}}=0$, as expected. Since the direction of the force represents the direction of initial motion and also since there is a fading memory, the particle shows an initial displacement and at large times the periodic motion manifests itself, approximately.

The mean particle velocity is very small and in regions of low $R e_{\mathrm{F}}$ and high $R e$ is in the direction of first motion of the particle. This is as expected because the periodic nature of the force dominates at long times. The initial shift before the particle settles down to an approximate oscillatory motion about a mean is determined by the initial motion. In the long time limit, the effect of the memory of the initial motion is manifested mainly on the position of the particle and not on the velocity of the particle. However, we observe that there is a small mean drift velocity of the order of $10^{-6}$ in certain regions of $R e$ and $R e_{\mathrm{F}}$. The relationship of $R e$ and $R e_{\mathrm{F}}$ with the amplitude of the velocity of the particle was obtained and this is shown in figure 11. This figure gives the behavior of the amplitude of velocity with respect to $R e$ and $R e_{\mathrm{F}}$. If we suppose that a periodic force is applied on the particle through an electric field, then the magnitude of the charge on the particle could be obtained by the known values of the electric field $E$, the Reynolds number $R e$ and $R e_{\mathrm{F}}$ and the plot of the amplitude of velocity, or any of the other plots presented here that show dependence of an observable property on $R e$ and $R e_{\mathrm{F}}$. We observe that the amplitude of velocity increases with an increase in $R e_{\mathrm{F}}$ and decreases with an increase in $R e$, describing the effect of the periodic force and the effect of inertia on the amplitude of the velocity of the particle. However, the plot of $\boldsymbol{U}_{\mathrm{p}}$ 


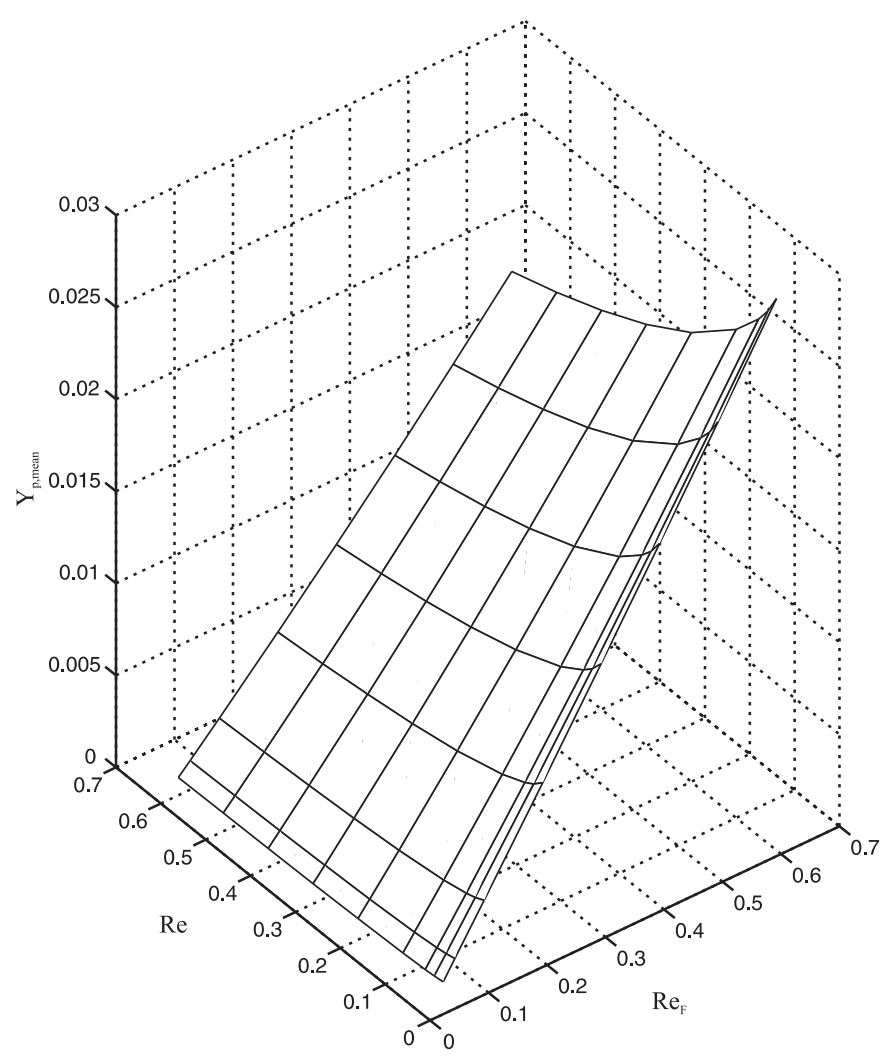

Figure 9. A surface plot showing the relationship of $Y_{\mathrm{p}, \text { mean }}$ with $R e$ and $R e_{\mathrm{F}}$.

amplitude against $R e$ and $R e_{\mathrm{F}}$ also shows a reflection property, increasing our confidence in our simulation.

We tested our solutions for chaos and found that there was no chaos in the system in the parametric regime that we have considered (Kantz and Schrieber 2005).

Our problem can be considered to consist of an excitable medium, as the periodic force when applied on the particle creates stresses in the fluid due to the motion of the fluid and particle. An analogy can be the effect of Brownian motion leading to a contribution to the stress tensor in a sheared suspension. The Brownian motion can be considered to be replaced by a periodic force in our problem. Thus, the only significant rheological parameter in the problem, namely a 'normal stress', was computed following Batchelor's technique (Batchelor 1970). Batchelor developed a method for computing the bulk stress generated by a flowing suspension in terms of volume averages. In this formulation we consider a suspension of particles in a quiescent Newtonian fluid. We assume that the particles are far enough from each other such that there is no interaction between the particles, i.e. the volume fraction $\phi$ is assumed to be small. Hence, the particle contribution to the bulk stress is given by Lin et al (1970), Kulkarni and Morris (2008) and Patankar and $\mathrm{Hu}$ (2002) at low Reynolds numbers. Since the particle motion is approximately oscillatory, we assumed that there was no net motion of the particle through the fluid. Under these assumptions, we obtain

$$
\Sigma^{p}=\frac{1}{V} \sum_{i} \boldsymbol{S}_{i}-\frac{R e}{V} \sum_{i} \int_{V_{\mathrm{p}}} \frac{1}{2}(\boldsymbol{a x}+\boldsymbol{x} \boldsymbol{a}) \mathrm{d} V_{i}-\frac{R e}{V} \int_{V} \boldsymbol{u}^{\prime} \boldsymbol{u}^{\prime} \mathrm{d} V
$$




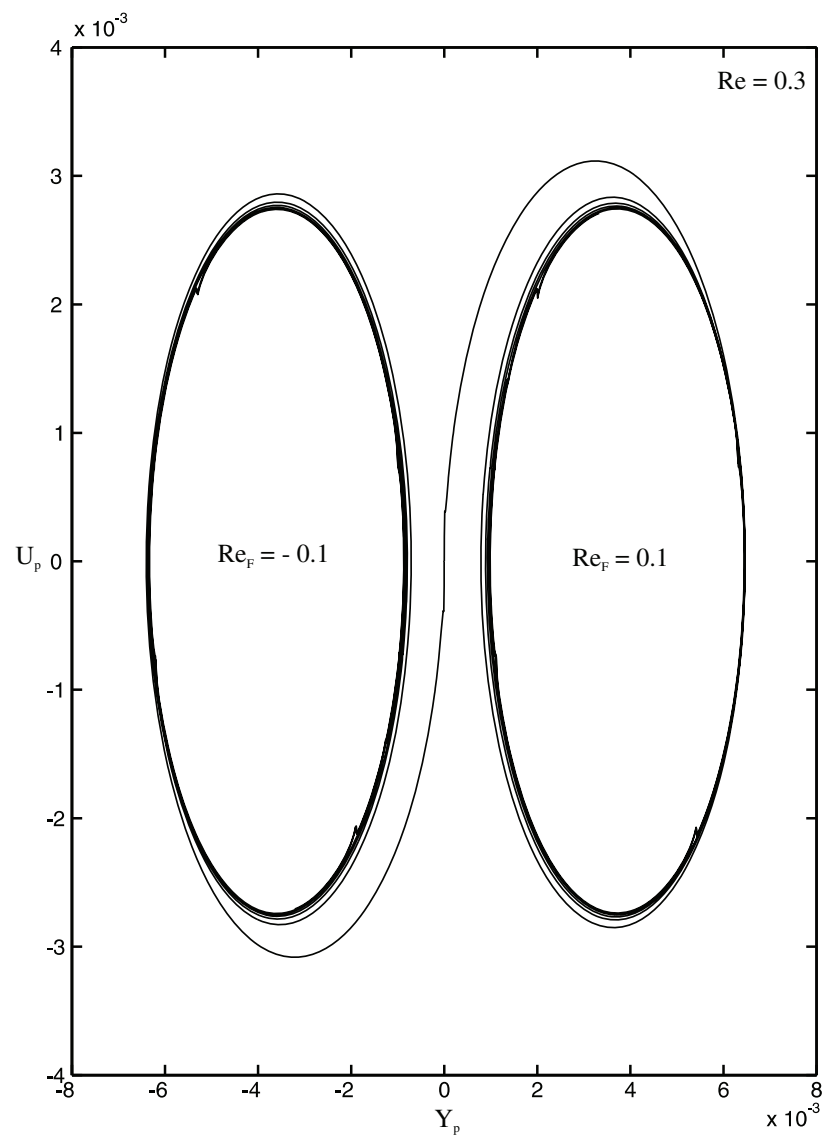

Figure 10. The phase portrait obtained at $R e=0.3$ and $R e_{\mathrm{F}}= \pm 0.1$. The phase portrait shows the reflection property of the solutions of our problem, indicating that there is a physical basis to our results.

Here, the first term represents the stresslet, or symmetric first moment of surface stress, exerted by a particle $i$, given for a rigid particle by

$$
S_{i}=\int_{A_{\mathrm{p}}} \frac{1}{2}(\boldsymbol{x} \boldsymbol{\sigma} \cdot \boldsymbol{n}+\boldsymbol{\sigma} \cdot \boldsymbol{n} \boldsymbol{x}) \mathrm{d} A_{i} .
$$

Here, $\boldsymbol{n}$ is the normal directed outward from the particle surface into the fluid phase. Following Brady and Bossis (1988), the expression for bulk stress at zero $R e$ is given as

$$
\left\langle\sum\right\rangle=\mathrm{IT}+2 \eta \boldsymbol{E}^{\infty}+\left(\frac{N}{V}\right)\left\{\left\langle\boldsymbol{S}^{\boldsymbol{H}}\right\rangle+\left\langle\boldsymbol{S}^{\boldsymbol{P}}\right\rangle+\left\langle\boldsymbol{S}^{\boldsymbol{B}}\right\rangle\right\} .
$$

Here, IT is an isotropic term.

$$
\boldsymbol{E}^{\infty}=\frac{1}{2}\left(\nabla \boldsymbol{u}^{\infty}+\nabla \boldsymbol{u}^{\infty^{T}}\right) .
$$

The above term is zero as $\boldsymbol{u}^{\infty}$ is a function of $t$ alone. $\boldsymbol{S}^{\boldsymbol{H}}$ is a mechanical or contact stress transmitted by the fluid due to shear flow. However, in our problem there is negligible shear and the value of this term is zero. $\left\langle\boldsymbol{S}^{\boldsymbol{P}}\right\rangle=-\left\langle\boldsymbol{x} \boldsymbol{P}^{\boldsymbol{P}}\right\rangle$ and $\boldsymbol{S}^{\boldsymbol{B}}$ is the direct contribution from the Brownian motion that is also zero for our problem. 


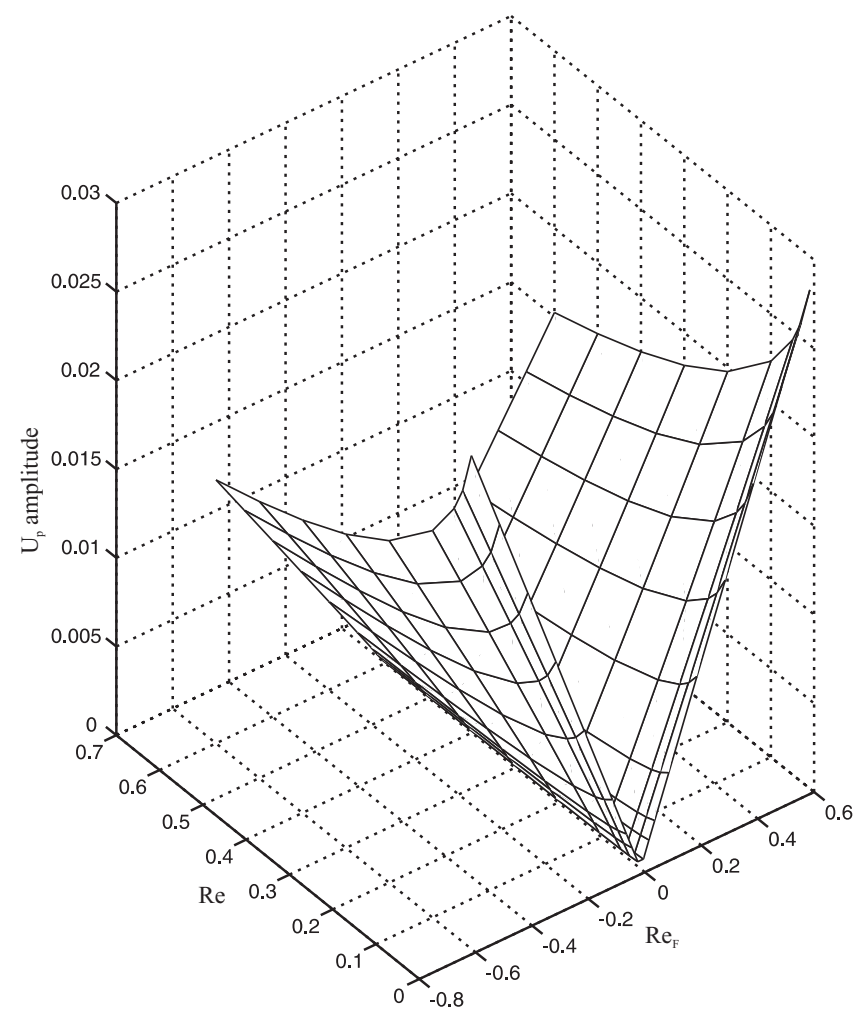

Figure 11. A surface plot of the amplitude of the particle velocity versus $R e$ and $R e_{\mathrm{F}}$.

Thus, for our problem bulk stress is given by

$$
\Sigma^{p}=-\left\langle\boldsymbol{x} \boldsymbol{F}^{\boldsymbol{P}}\right\rangle-\frac{R e}{V} \sum_{i} \int_{V_{\mathrm{p}}} \frac{1}{2}(\boldsymbol{a x}+\boldsymbol{x} \boldsymbol{a}) \mathrm{d} V_{i}-\frac{R e}{V} \int_{V} \boldsymbol{u}^{\prime} \boldsymbol{u}^{\prime} \mathrm{d} V .
$$

The first term is the average of the product of the external force on the particle and displacement of the particle. The second and the third terms in the expression (5.5) of the particle stress denote the stress due to acceleration of the particles and the Reynolds stress, respectively. The expression (5.5) directly shows that the contributions to the bulk stress from the acceleration and Reynolds stress are dependent on $R e$; the stresslet is dependent on $R e$ through the flow field. For a dilute suspension, the interactions between the particles can be neglected and the solution for a single particle can be used to calculate the particle stress. Further, for our problem, the force is the external force $F_{\text {ext }}$ and the displacement vector is $\left(x_{0}+Y_{\mathrm{p}}, y_{0}, z_{0}\right)$ for a particle. Here, $\left(x_{0}, y_{0}, z_{0}\right)$ are the coordinates of the rest position of one particle. Since the particle displacement $Y_{\mathrm{p}}$ isonly along the $x$-axis, only $\sum_{x x}^{p}$ is significant. All the other components of the stress tensor will be negligible when the mean position of the suspension is set to zero. We note here that when the initial displacement of the particle is changed, the attractor just changes its coordinates and no other change is observed in the attractor. Hence, to calculate the normal stress, we can choose $\Sigma x_{0}=0$; similarly, $\Sigma y_{0}=\Sigma z_{0}=0$ over the position of the particles. That is, we choose the mean displacement of the averages of the rest position of the particles to be zero. The evaluation of the third term on the rhs of equation (5.1) which at least, in principle, contributes non-zero terms to the other components of the stress tensor is shown below to be negligible. 


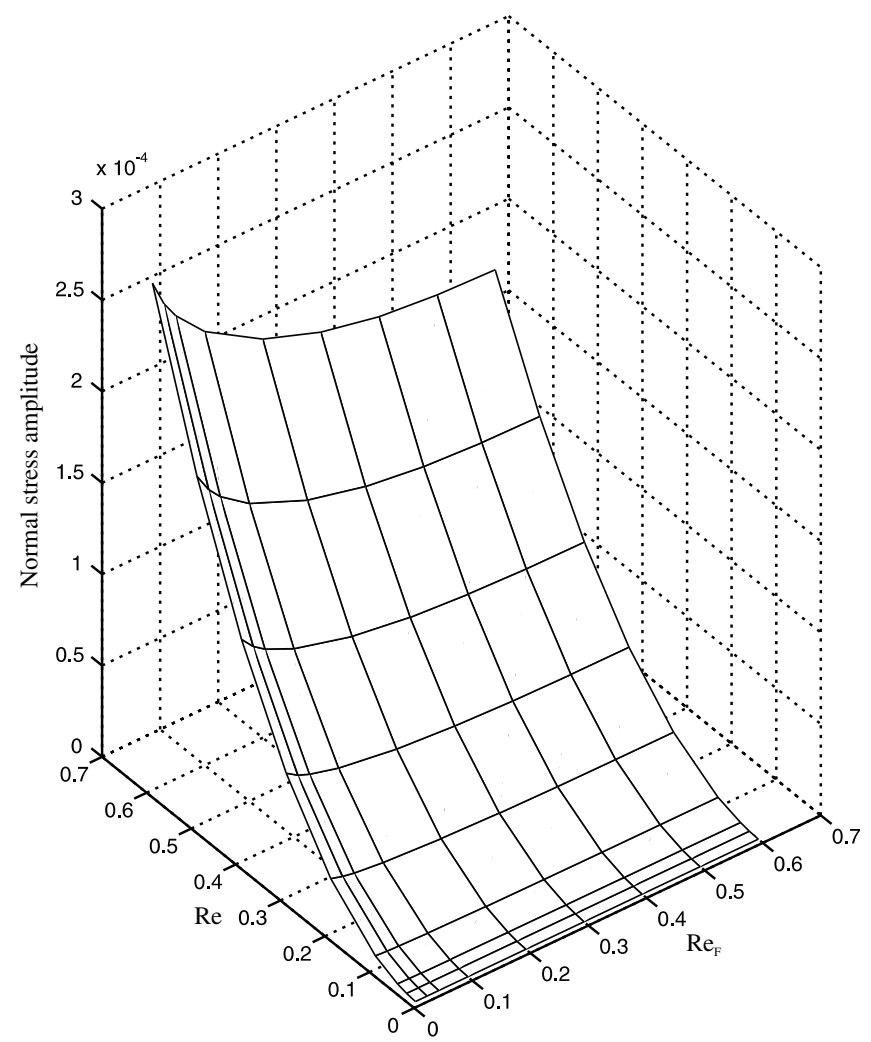

Figure 12. Surface plots showing the relationship of the amplitudes of the normal stress with $R e$ and $R e_{\mathrm{F}}$.

\section{Calculation of the integral $\int_{V} \boldsymbol{u}^{\prime} \boldsymbol{u}^{\prime} \mathrm{d} V$}

In spherical coordinates, we have the velocity components for a flow past a moving sphere as:

$$
\begin{aligned}
& v_{r}=-\frac{1}{2} U \cos \theta\left(\frac{1}{r}\right)^{2}\left(\frac{1}{r}-3 r\right), \\
& v_{\theta}=-\frac{1}{4} U \sin \theta\left(\frac{1}{r}\right)\left[\left(\frac{1}{r}\right)^{2}+3\right], \\
& v_{\varphi}=0 .
\end{aligned}
$$

In the Cartesian coordinate system, we obtain

$$
\begin{aligned}
& v_{x}=\frac{3 U x z}{4 r^{5}}\left(r^{2}-1\right), \\
& v_{y}=\frac{3 U y z}{4 r^{5}}\left(r^{2}-1\right), \\
& v_{z}=\frac{U}{4 r}\left(\frac{3 z^{2}}{r^{2}}-\frac{3 z^{2}}{r^{4}}+3+\frac{1}{r^{2}}\right),
\end{aligned}
$$

where $r=\sqrt{x^{2}+y^{2}+z^{2}}$ and $\boldsymbol{U}=\boldsymbol{U}_{\mathrm{p}}$.

Hence, we obtain $\boldsymbol{u}^{\prime}=\left(v_{x}, v_{y}, v_{z}\right)$. 


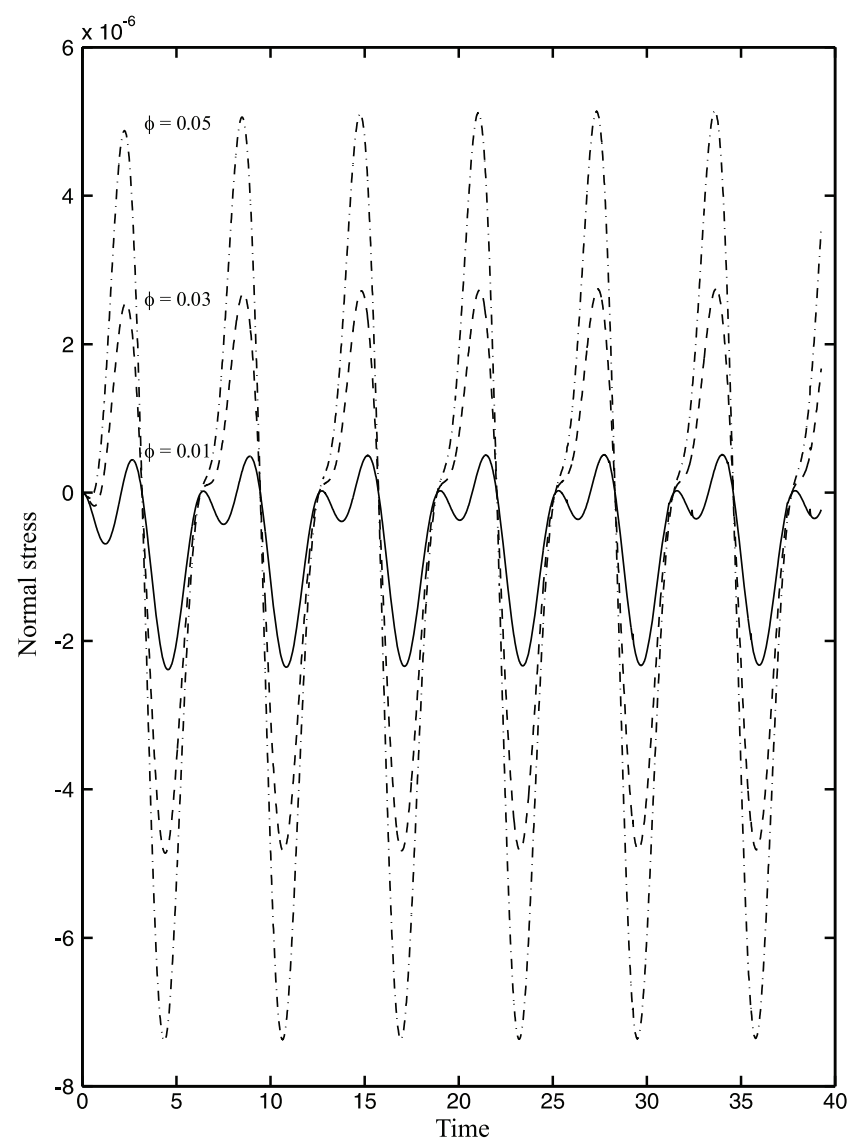

Figure 13. Relationship between mean normal stress and different volume fractions.

We took the tensor product $\boldsymbol{u}^{\prime} \boldsymbol{u}^{\prime}$ after removing the contribution due to the Stokeslet, since the effect of the force field on the stress tensor has been included in the first term in equation (5.1), and obtained nine components which are integrated in the Cartesian coordinate system. We performed the integration using the limit of a sum concept for the triple integral using Matlab. The limit upon $\boldsymbol{U}_{\mathrm{p}}$ was considered and the diminishing of $\boldsymbol{u}^{\prime} \boldsymbol{u}^{\prime}$ was evident during the integration as $(x, y, z)$ tends to infinity. The numerical integration was performed by taking the step size $h=0.01$ and $x, y$ and $z$ varying from 1 to 10 . Further change in the parameters did not affect the values of the integral up to four significant digits.

Thus, the components of the particle stress are found to be

$$
\begin{aligned}
\Sigma_{x x}^{p}= & \frac{3}{4 \pi} \varphi Y_{\mathrm{p}}(t) \operatorname{Re}_{F} \sin (t)-\frac{\operatorname{ReRe}_{\mathrm{F}}^{2} \varphi \cos (t)(1-\cos (t))}{36 \pi^{2}} \\
& -2.07 \operatorname{Re}_{\mathrm{p}}^{2}\left(10^{-10}\right), \\
\Sigma_{x y}^{p}= & \Sigma_{y x}^{p}=\Sigma_{y y}^{p}=-2.07 \operatorname{Re}_{\mathrm{p}}^{2}\left(10^{-10}\right) \sim 0, \\
\Sigma_{x z}^{p}= & -8.3056 \operatorname{Re} U_{\mathrm{p}}^{2}\left(10^{-10}\right) \sim 0, \\
\Sigma_{x z}^{p}= & \Sigma_{z x}^{p}=\Sigma_{z y}^{p}=\Sigma_{y z}^{p}, \\
\Sigma_{z z}^{p}= & -3.33 \operatorname{Re} U_{\mathrm{p}}^{2}\left(10^{-9}\right) \sim 0 .
\end{aligned}
$$


Hence, the only significant term is $\Sigma_{x x}^{p}$. This is correct only to $\mathrm{O}(R e)$. We have not considered higher orders of $R e$ in the problem as our equations are valid only up to $\mathrm{O}(R e)$. For the Stokes' flow case the stress in the suspension is given by

$$
\Sigma_{x x}^{p}=\frac{3}{4 \pi} \varphi R e_{\mathrm{F}}^{2} \sin (t)(1-\cos (t)) .
$$

We varied $\phi, R e$ and $R e_{\mathrm{F}}$ and studied their effect on the normal stress. As $\phi$ increases, the normal stress increases, and the normal stress also increases with an increase in both $R e$ and $R e_{\mathrm{F}}$. Figure 12 shows the relationship of the amplitudes of the normal stress with $R e$ and $R e_{\mathrm{F}}$. Figure 13 shows its relationship with $\phi$. The normal stress can be measured more easily than the motion of the particle and hence it can be more easily correlated to system parameters.

The presence of a normal stress in the dilute suspension is evidence for non-Newtonian behavior. Hence, this system exhibits non-Newtonian rheology.

The strong relationship between $R e, R e_{\mathrm{F}}$ and normal stress, evident from figure 13, makes our results interesting, as we see that small changes in controllable parameters lead to relatively large changes in the rheological parameters. Here, we observe that the amplitude of the normal stress is approximately linearly dependent on $R e_{\mathrm{F}}$ at low $R e$, and as $R e$ increases, the amplitude of the normal stress increases more rapidly with $R e_{\mathrm{F}}$. Similarly, at low $R e_{\mathrm{F}}$, the amplitude of the normal stress increases slowly with $R e$, and at high $R e_{\mathrm{F}}$, the amplitude of the normal stress increases rapidly with $R e$.

\section{Conclusions}

In this paper, we have attempted to determine the effects of a periodic force on a sphere in a quiescent fluid at low Reynolds numbers. We observe that the particle oscillates around a mean position, due to the periodic force on the particle. There is a net displacement of the mean position of the particle in the direction of the first motion. We have compared the results obtained in our simulations with the results of Stokes' flow. We set the nonlinear term in our equations to be equal to zero and compared our results with those solutions. It is observed that an increase in $R e$ was responsible for an increase in the resistance to change in particle motion and hence a decrease in attractor area and an increase in $R e_{\mathrm{F}}$ lead to an increase in the amplitude of motion of the particle. In addition, a reflection of the attractor about the position axis is obtained by changing the initial direction of motion. Our results on the dependence of the mean position of the particle and the amplitude of the velocity of a particle on the problem parameters, such as $R e$ and $R e_{\mathrm{F}}$, may be used to estimate appropriate physical parameters of the system by suitable experiments. We have also shown here that there is a strong direct relationship between a rheological parameter and physical parameters such as $R e$ and $R e_{\mathrm{F}}$. Thus, we have shown that the rheology of the system can be controlled by changing the physical parameters, which may be a technologically important result. We have presented physical arguments that support our results. We also note that the rheology of suspensions of such particles exhibits non-Newtonian behavior. We further note that this is a physically realizable system that can be used to probe the relation between the dynamics of individual oscillators with memory and the methods used to derive macroscopic averages from them. We hope that this work will excite further interest in this area.

\section{Acknowledgments}

We thank Dr A R Upadhya, Scientist-in-Charge, CSIR CMMACS, Bangalore, India, and Professor N Rudraiah, Honorary Professor, UGC CAS, Department of Mathematics, 
Bangalore University, Bangalore, India, for their constant encouragement. We acknowledge the Department of Science and Technology, Government of India, New Delhi, India, for financial support vide Sanction Letter No. SR/S3/CE/33/2004-SERC Engg. KM acknowledges NAL/C-MMACS for support through a fellowship. We thank the Editor and the referees for their comments which have helped us to improve this paper substantially.

\section{References}

Abbad M and Souhar M 2004 Effects of the history force on an oscillating rigid sphere at low Reynolds numbers Exp. Fluids 36775

Asokan K, Kumar C V A, Dasan J, Radhakrishnan K, Kumar K S and Ramamohan T R 2005 Review of chaos in the dynamics and rheology of suspensions of orientable particles in simple shear flow subjected to an external periodic force J. Non-Newtonian Fluid Mech. 129128

Batchelor G K 1970 The stress system in a suspension of force-free particles J. Fluid Mech. 41545

Becker L E, Mckinley G H and Stone H A 1996 Sedimentation of a sphere near a plane wall: weak non-Newtonian and inertial effects J. Non-Newtonian Fluid Mech. 63201

Brady J F and Bossis G 1988 Stokesian dynamics Ann. Rev. Fluid Mech. 20111

Chang E J and Maxey M R 1994 Unsteady flow about a sphere at low to moderate Reynolds numbers, part 1: oscillatory motion J. Fluid Mech. 277347

Dasan J, Ramamohan T R, Singh A and Nott P R 2002 Stress fluctuations in sheared Stokesian suspensions Phys. Rev. E 66021409

Jansons K M 2007 Stochastic Stokes' drift with inertia Proc. R. Soc. A 463521

Kantz H and Schrieber T 2005 Nonlinear Time Series Analysis 2nd edn (Cambridge: Cambridge University Press)

Kim J M and Phillips R J 2004 Dissipative particle dynamics simulation of flow around spheres and cylinders at finite Reynolds numbers Chem. Eng. Sci. 59 4155-68

Kulkarni P M and Morris J F 2008 Suspension properties at finite Reynolds number from simulated shear flow Phys. Fluids 20040602

Kumar K S and Ramamohan T R 1995 Chaotic rheological parameters of periodically forced slender rods in simple shear flow J. Rheol. 391229

Kumar K S, Savithri S and Ramamohan T R 1999 Review of chaotic behavior of suspensions of slender rods in simple shear flow Dynamics of Complex Fluids ed M J Adams, A R Rennie, R A Mashelkar and J R A Pearson (London: Imperial College Press, The Royal Society) pp 286-300

Leshansky A M and Brady J F 2004 Force on a sphere via the generalized reciprocal theorem Phys. Fluids 16843

Leshansky A M, Lavrenteva O M and Nir A 2004 The leading effect of fluid inertia on the motion of rigid bodies at low Reynolds numbers J. Fluid Mech. $\mathbf{5 0 5} 235$

Lin C J, Perry J H and Schowalter W R 1970 Simple shear flow round a rigid sphere: inertial effects and suspension rheology J. Fluid Mech. 441

Lovalenti P M and Brady J F 1993 The hydrodynamic force on a rigid particle undergoing arbitrary time-dependent motion at small Reynolds number J. Fluid Mech. 256561

Mikulencak D R and Morris J F 2004 Stationary shear flow around fixed and free bodies at finite Reynolds numbers J. Fluid Mech. 000 1-28

Patankar N A and Hu H H 2002 Finite Reynolds number effect on a rheology of a dilute suspension of neutrally buoyant circular particles in a Newtonian fluid Int. J. Multiph. Flows 28409

Press W H, Vetterling W T, Teukolsky S A and Flannery B P 1992 Numerical recipes in FORTRAN 77 The Art of Scientific Computing 2nd edn (Cambridge: Cambridge University Press)

Radhakrishnan K, Asokan K, Dasan J, Bhat C C and Ramamohan T R 1999 Numerical evidence for the existence of a low-dimensional attractor and its implications in the rheology of dilute suspensions of periodically forced slender bodies Phys. Rev. E 606602

Roscoe R 1967 On the rheology of a suspension of viscoelastic spheres in a viscous liquid J. Fluid Mech. 28273

Stokes G G 1851 On the effect of internal friction of fluid on the motion of pendulums Trans. Camb. Phil. Soc. 98 